\title{
Novel immunophenotypic features of marrow lymphoplasmacytic lymphoma and correlation with Waldenström's macroglobulinemia
}

\author{
William G Morice, Dong Chen, Paul J Kurtin, Curtis A Hanson and Ellen D McPhail \\ Division of Hematopathology, Department of Laboratory Medicine and Pathology, Mayo Clinic, Rochester, \\ $M N, U S A$
}

\begin{abstract}
Lymphoplasmacytic lymphoma involving the bone marrow can be difficult to diagnose, and pathological features that predict the presence of associated Waldenström's macroglobulinemia have yet to be identified. To address these issues, marrow histology, immunohistochemistry, and flow cytometry were studied from 35 lymphoplasmacytic lymphoma cases that had comprehensive clinical assessment for Waldenström's macroglobulinemia. In all cases, the plasma cells were analyzed by a novel 6-color flow method. Both immunohistochemistry and flow cytometry were useful in identifying the lymphoid and plasmacytic disease components. In 19 cases, immunohistochemistry revealed an earlier unrecognized pattern of plasma cell infiltration in which they were physically separate from the lymphoid infiltrates. B-cell flow cytometry revealed monotypic cells in $96 \%$ of the cases. Approximately half of these were CD5 and/or CD23 positive, although none had features of chronic lymphocytic leukemia, and none of the B cells had flow cytometric features suggesting plasmacytic differentiation. In contrast, highly sensitive 6-color plasma cell flow cytometry revealed monotypic cells in 32 of the 35 cases; in 20 cases, the pattern of CD38 and CD138 coexpression detected was identical to that seen in plasma cell malignancies such as multiple myeloma. In 18 of these 20 lymphoplasmacytic lymphoma cases, these plasma cells were CD19 positive, distinguishing them from those of true plasma cell neoplasms, which are CD19 negative. It is interesting that the two lymphoplasmacytic lymphoma cases with CD19-negative plasma cells had an IgG isotype serum paraprotein. Apart from this, no other pathological correlates of the clinical or laboratory features of symptomatic Waldenström's macroglobulinemia were identified.

Modern Pathology (2009) 22, 807-816; doi:10.1038/modpathol.2009.34; published online 13 March 2009
\end{abstract}

Keywords: lymphoplasmacytic lymphoma; Waldenström's macroglobulinemia; flow cytometry; bone marrow; immunohistochemistry

Lymphoplasmacytic lymphoma is a low-grade B-cell malignancy exhibiting a cytological spectrum of plasmacytic differentiation ranging from small lymphocytes to true plasma cells, and including cells with features intermediate between these two, referred to as plasmacytoid lymphocytes. ${ }^{1}$ Lymphoplasmacytic lymphoma characteristically has an associated monoclonal serum immunoglobulin $\mathrm{M}$ (IgM) paraprotein, although neither the paraprotein isotype nor the quantity reliably distinguishes lymphoplasmacytic lymphoma from other lymphoma types. ${ }^{2}$ As lymphoplasmacytic lymphoma infrequently involves the lymph nodes or other extramedullary sites, bone marrow biopsy is often

Correspondence: Dr WG Morice, MD, PhD, Division of Hematopathology, Department of Laboratory Medicine and Pathology, Mayo Clinic, 200 1st St. SW, Rochester, MN 55905, USA.

E-mail: morice.william@mayo.edu

Received 15 December 2008; revised and accepted 20 February 2009; published online 13 March 2009 the first and only diagnostic tissue obtained, and therefore recognition of its manifestations in this site is critically important. Unfortunately, given the histological overlap between lymphoplasmacytic lymphoma and other mature B-cell malignancies with plasmacytic differentiation, such as marginal zone B-cell lymphoma, when the pathologist encounters these neoplasms, a generic diagnosis of 'low-grade B-cell malignancy with plasmacytic differentiation' is often rendered and further subclassification is deferred to correlation with clinical features. $^{3}$

In a subset of lymphoplasmacytic lymphoma patients, the paraproteinemia is associated with distinctive clinical findings, such as serum hyperviscosity, cryoglobulinemia, autoimmune phenomena such as peripheral neuropathy, and amyloidosis. On the basis of early descriptions by Waldenström of this syndromic presentation of paraproteinemia and associated clinical features, the term Waldenström's macroglobulinemia was coined. ${ }^{4}$ The close 
relationship between these entities has led to these terms often being used synonymously, although features of Waldenström's macroglobulinemia are not uniformly present in lymphoplasmacytic lymphoma. $^{3}$ The diversity of clinical findings in Waldenström's macroglobulinemia has in turn led to variability in the attributes of patients who ascribed to this diagnosis. To address this issue, an internationally recognized group of field experts met and reached a consensus on the defining clinicopathological features of Waldenström's macroglobulinemia. ${ }^{5}$ According to this consensus, bone marrow involvement in lymphoplasmacytic lymphoma is a requisite minimal diagnostic criterion. Patients are further subdivided into symptomatic or asymptomatic Waldenström's macroglobulinemia on the basis of the presence or absence of clinical findings attributable to either organ infiltration by the tumor or effects of the serum paraprotein noted above. Although marrow examination plays a central role in making the diagnosis, there have been relatively few studies of the histopathology of lymphoplasmacytic lymphoma in this site and fewer still selectively analyzing the immunophenotype of the lymphocytic and plasmacytic components. ${ }^{1,6,7}$ Furthermore, the identification of pathological features in lymphoplasmacytic lymphoma, which might correlate with either the amount of IgM paraproteinemia or the clinical findings of Waldenström's macroglobulinemia, has remained elusive. $^{8}$

Recent advances in flow cytometry have allowed for the introduction into the clinical laboratory of powerful multicolor approaches to plasma cell evaluation. In these analyses, plasma cells are identified through CD38 and CD138 coexpression, and normal and abnormal cells are distinguished through the differential expression of CD19 and CD45 in conjunction with the documentation of cytoplasmic Ig light chain restriction. ${ }^{9,10}$ In this study, the results of these high power plasma cell flow cytometric analyses were compared with the bone marrow aspirate and biopsy pathology, immunohistochemistry, B-cell flow cytometric immunophenotyping, and with the serological and clinical features in a group of 35 lymphoplasmacytic lymphoma cases. The goals of this study were to fully characterize the pathological features of marrow involvement by lymphoplasmacytic lymphoma, to determine whether there were pathological features that correlated with the serological and/ or clinical findings of Waldenström's macroglobulinemia, and to develop a rational approach to using these tools for diagnosing marrow involvement by lymphoplasmacytic lymphoma.

\section{Materials and methods}

Clinical and laboratory data from 35 patients in whom bone marrow examination was carried out over a 12-month period at the Mayo Clinic Rochester as part of the evaluation for IgM paraproteinemia, possible lymphoplasmacytic lymphoma, or possible Waldenström's macroglobulinemia were reviewed. In all cases, a diagnosis of plasma cell malignancy was clinically excluded; a single case with an IgM paraprotein was not included in this study because the clinical and radiological features raised the possibility of multiple myeloma. The bone marrow aspirates and biopsies were all reviewed. Paraffin immunohistochemistry was carried out using an earlier described methodology in 27 cases with antibodies to the following: CD20 $(n=25$, clone: L26, commercial source: Dako, Carpinteria, CA, USA), CD138 $(n=25$, clone M115, Dako), PAX5 $(n=14$; clone 24 , BD Bioscience, San Jose, CA, USA), $\kappa$ and $\lambda$ cytoplasmic Ig light chains $(n=17$, polyclonal, Dako), and CD79a $(n=3$, clone JCB117, Dako). ${ }^{11}$ Only 2 of the 35 patients had documented extramedullary tissue involvement, in keeping with the typical attributes of lymphoplasmacytic lymphoma. In one case, splenectomy carried out for the treatment of hemolytic anemia revealed minimal white-pulp infiltrates consistent with lymphoplasmacytic lymphoma, and, in the other, lymph node biopsy revealed medullary infiltrates with features diagnostic of lymphoplasmacytic lymphoma.

Plasma cell flow cytometric analysis was carried out on all 35 bone marrow aspirates by an earlier published single-tube, 6-color method, using antibodies to CD19, CD38, CD45, CD138, and $\kappa$ and $\lambda$ cytoplasmic Ig light chains. ${ }^{10}$ These data were acquired on an FACSCanto instrument (BD Biosciences) and analyzed using BD FACSDiva software. 4-color B-lymphocyte flow cytometry was also carried out in 27 cases using mononuclear cells isolated from either the marrow aspirate $(n=26)$ or peripheral blood $(n=1)$, and fluorochromeconjugated antibodies to CD19, CD10, CD45, and $\kappa$ and $\lambda$ surface Ig light chains. In 23 of these 27 cases, additional flow cytometry was carried out with fluorochrome-conjugated antibodies to CD5, CD11c, CD22, CD23, CD38, and CD103. Earlier published methods were used for the B-lymphocyte flow cytometry, with BD FACSCalibers for data acquisition and BD CellQuest Pro software for data analysis. ${ }^{12}$

This study was approved by the Mayo Clinic Rochester IRB, and all the participants consented to the review of their medical record for research purposes.

\section{Results}

The study group included 21 men and 14 women with a median age of 68 years (range: 42-93); pertinent clinical and laboratory features are shown in Table 1. A total of 29 of the 35 cases (83\%) met the published diagnostic criteria for symptomatic Waldenström's macroglobulinemia based on the 
Table 1 Clinical and laboratory features in the lymphoplasmacytic lymphoma cases

\begin{tabular}{lccccc}
\hline $\begin{array}{l}\text { IgM paraprotein } \\
(\%)^{\mathrm{a}}\end{array}$ & $\begin{array}{c}\text { IgG or IgA } \\
\text { paraprotein }(\%)^{\mathrm{b}}\end{array}$ & $\begin{array}{c}\text { M-spike median } \\
(\text { range) }\end{array}$ & $\begin{array}{c}\text { Increased serum } \\
\text { viscosity }(\%)\end{array}$ & $\begin{array}{c}\text { Cryoglob }^{\mathrm{d}} \\
(\%)\end{array}$ & $\begin{array}{c}\text { Peripheral } \\
\text { neuropathy }\end{array}$ \\
\hline $32(91 \%)$ & $6(17 \%)$ & $1.5 \mathrm{~g}$ per $100 \mathrm{ml}(0.3-5.1)$ & $11(55 \%)$ & $3(30 \%)$ & $6(17 \%)$ \\
\hline
\end{tabular}

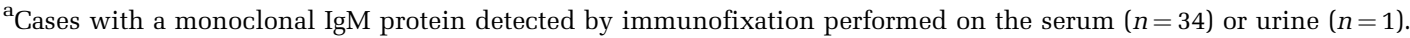

${ }^{\mathrm{b}}$ Cases with a monoclonal $\operatorname{IgG}(n=5)$ or $\operatorname{IgA}(n=1)$ protein by serum immunofixation. In four cases with an IgG monoclonal protein, an IgM monoclonal protein was also present.

${ }^{\mathrm{C}}$ Increased serum viscosity assessed in 20 cases.

${ }^{\mathrm{d}}$ Detectable serum cryoglobulin present assessed in 10 cases.

${ }^{\mathrm{e}}$ Cases with peripheral neuropathy documented by neurologic examination.

${ }^{\mathrm{f}}$ Cases with biopsy-proven amyloid deposition.

presence of one or more clinical features shown in the Table $1 .^{5}$

In all cases, the bone marrow was involved by lymphoplasmacytic lymphoma, and all four recognized patterns of infiltration (nodular, interstitial, paratrabecular, and diffuse) were represented (Figure 1, Table 2). ${ }^{1,6,7}$ All of the infiltrates contained plasma cells, and although estimating their percentage was challenging, given the cytological continuum from lymphocytes to plasma cells typical of lymphoplasmacytic lymphoma, the marrow aspirate and biopsy plasma cell percentages were within $5 \%$ of each other in 30 of the 35 cases. In most cases, plasma cells represented a minor component of the neoplastic infiltrate, although their proportion varied considerably between cases. Moreover, in seven $(20 \%)$ cases, plasma cells were the predominant histologically recognized cell type in either the bone marrow aspirate alone $(n=4)$ or in both the aspirate and biopsy specimens $(n=3)$. Plasma cells with intranuclear inclusions (Dutcher bodies) were present in 19 cases (54\%).

Immunohistochemistry carried out on 27 of the bone marrow biopsies uniformly revealed lymphocytic infiltrates that were positive for one or more of the B-cell-associated antigens: CD20, PAX5, and CD79a. In all, 12 of 25 cases (48\%) stained for CD20 showed the weak positivity described earlier in lymphoplasmacytic lymphoma, particularly when compared with CD79a and PAX5 (Figure 2a). ${ }^{1}$ Immunohistochemistry for CD138 and Ig light chains highlighted the presence of plasma cells in all cases tested (Figure 2b). The proportion of plasma cells estimated by immunohistochemistry was equal to, or slightly greater than, that estimated by biopsy histology in all but three cases (Table 2). When comparing the proportion of the lymphocytic and plasmacytic components of the infiltrates as estimated by immunohistochemistry, the plasma cells appeared equal to $(n=4)$ or greater than $(n=5)$ the B cells in nine cases. Most of these cases had a relatively high proportion of plasma cells (compared withthat of lymphocytes) by morphological review, but eight of the nine also exhibited weak CD20 immunoreactivity, which likely contributed to the underestimation of the lymphoid
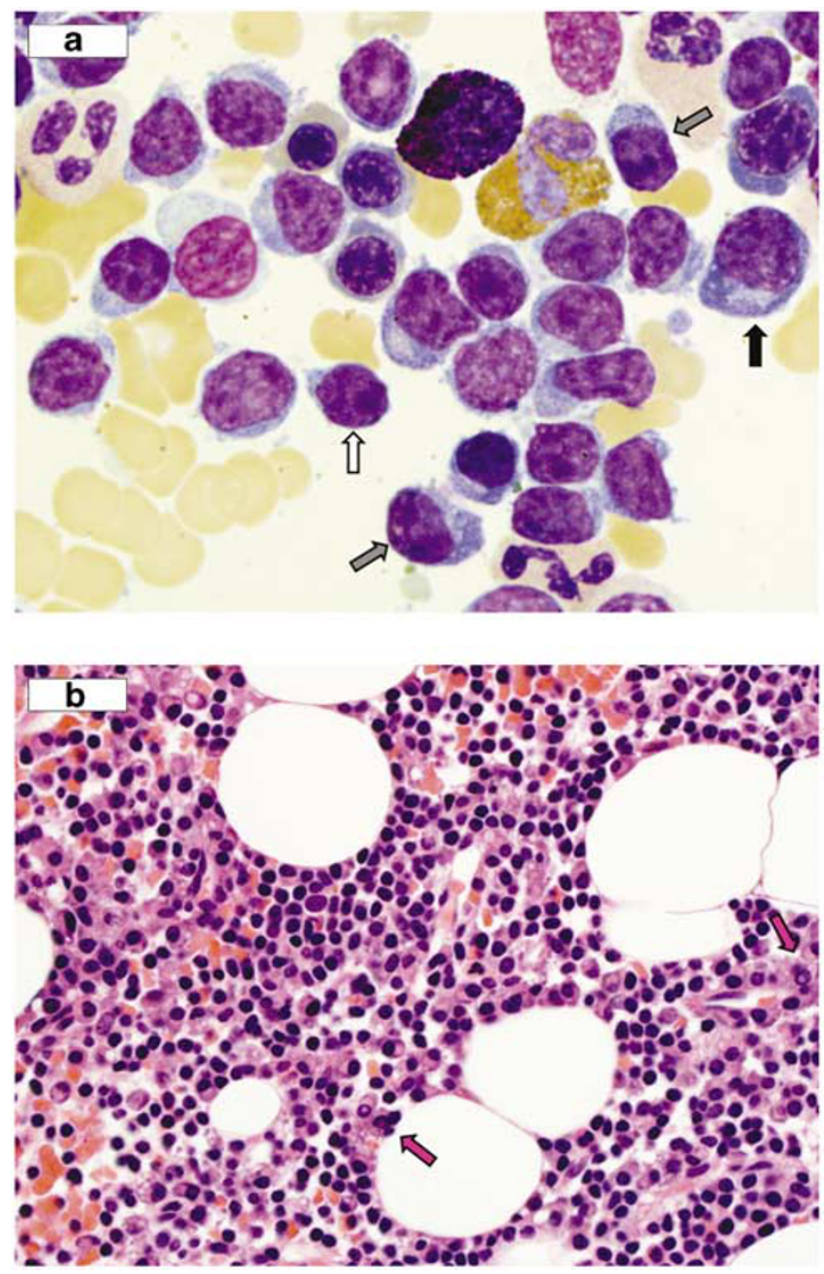

Figure 1 Marrow histopathology. (a) Plasma cells (black arrow), small lymphocytes (white arrow), and plasmacytoid lymphocytes with small eccentric nuclei and relatively abundant cytoplasm (gray arrows) in the marrow aspirate $(\times 1000$, oil). (b) Interstitial marrow infiltration by small lymphocytes and admixed plasma cells, some of which have Dutcher bodies (arrows; $\times 400$, oil).

component (compare Figure 2a and b). Both the lymphocytic and plasmacytic components were positive for PAX5 and CD79a, with some variability in PAX5 reactivity (Figure 2c). 
Table 2 BM features in the Waldenström's macroglobulinemia/lymphoplasmacytic lymphoma cases

\begin{tabular}{|c|c|c|c|c|c|}
\hline $\begin{array}{l}\% B M \text { involvement } \\
\text { median (range) }\end{array}$ & $\begin{array}{c}B M \text { distribution } \\
(\%)^{\mathrm{b}}\end{array}$ & $\begin{array}{c}\% P C s \text { BM aspirate } \\
\text { median (range) }\end{array}$ & $\begin{array}{l}\% \text { PCs BM biopsy } \\
\text { median (range) }\end{array}$ & $\begin{array}{l}\% P C s \text { by } C D 138 \\
\text { median (range) }\end{array}$ & $\begin{array}{l}\% \text { PCs by FCIP } \\
\text { median (range) }\end{array}$ \\
\hline $30(5-90)$ & $\begin{array}{l}\text { Nod: } 19(54) \\
\text { Int: } 29(83) \\
\text { ParaT: } 11(31) \\
\text { Diff: } 1(3)\end{array}$ & $4(1-26)$ & $5(1-30)$ & $10(5-50)$ & $0.7(0.1-4.6)$ \\
\hline
\end{tabular}

BM, bone marrow; Nod, nodular; Int, interstitial; ParaT, paratrabecular; Diff, diffuse

${ }^{\mathrm{a}}$ Percentage of BM involvement by lymphoplasmacytic lymphoma by morphologic review.

${ }^{\mathrm{b}}$ Distribution of infiltrates in BM.

${ }^{\mathrm{C}}$ Percentage of plasma cells in marrow aspirate by manual differential.

${ }^{\mathrm{d}}$ Percentage of plasma cells in marrow biopsy estimated by morphologic review.

${ }^{\mathrm{e}}$ Percentage of plasma cells in marrow biopsy estimated by immunoperoxidase staining for CD138.

fercentage of plasma cells in aspirate as detected by a 6-color flow cytometry.
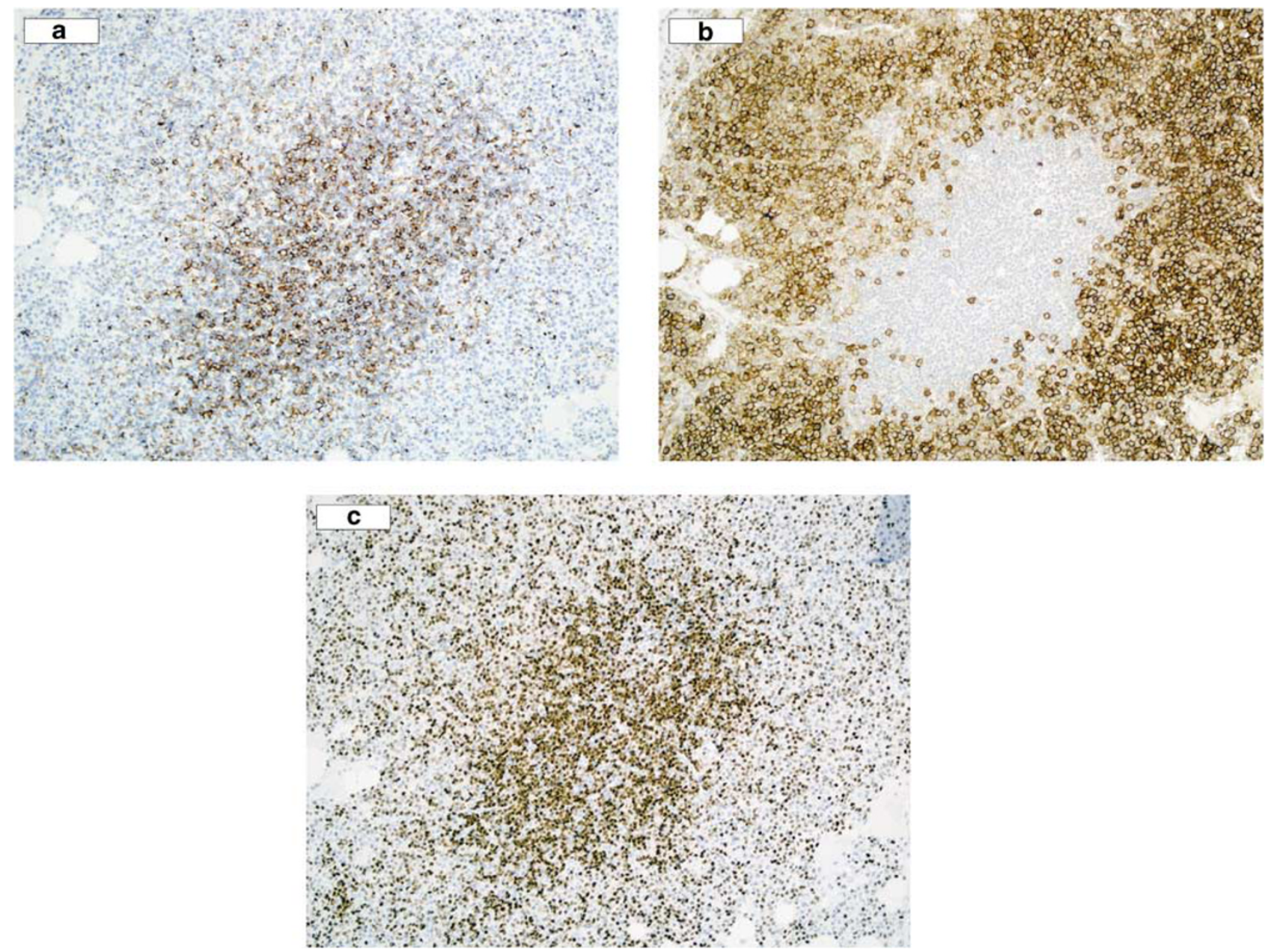

Figure 2 Marrow immunohistology. Immunohistochemistry for CD20, CD138, and PAX5 in a nodular lymphoid infiltrate reveals a center populated by weakly CD20-positive B cells (a) surrounded at the periphery by strongly CD138-positive plasma cells (b). The B cells and a subset of the plasma cells are PAX-5 positive (c).

The plasma cells identified by immunohistochemistry were associated with the discrete lymphoplasmacytic infiltrates in 25 of the 27 cases. These plasma cells were either admixed with the lymphocytes singly and in aggregates or arrayed in clusters and sheets at the periphery of lymphoid nodules (the latter shown in Figure $2 \mathrm{~b}$ ). In addition, in 19 cases, immunohistochemistry highlighted 

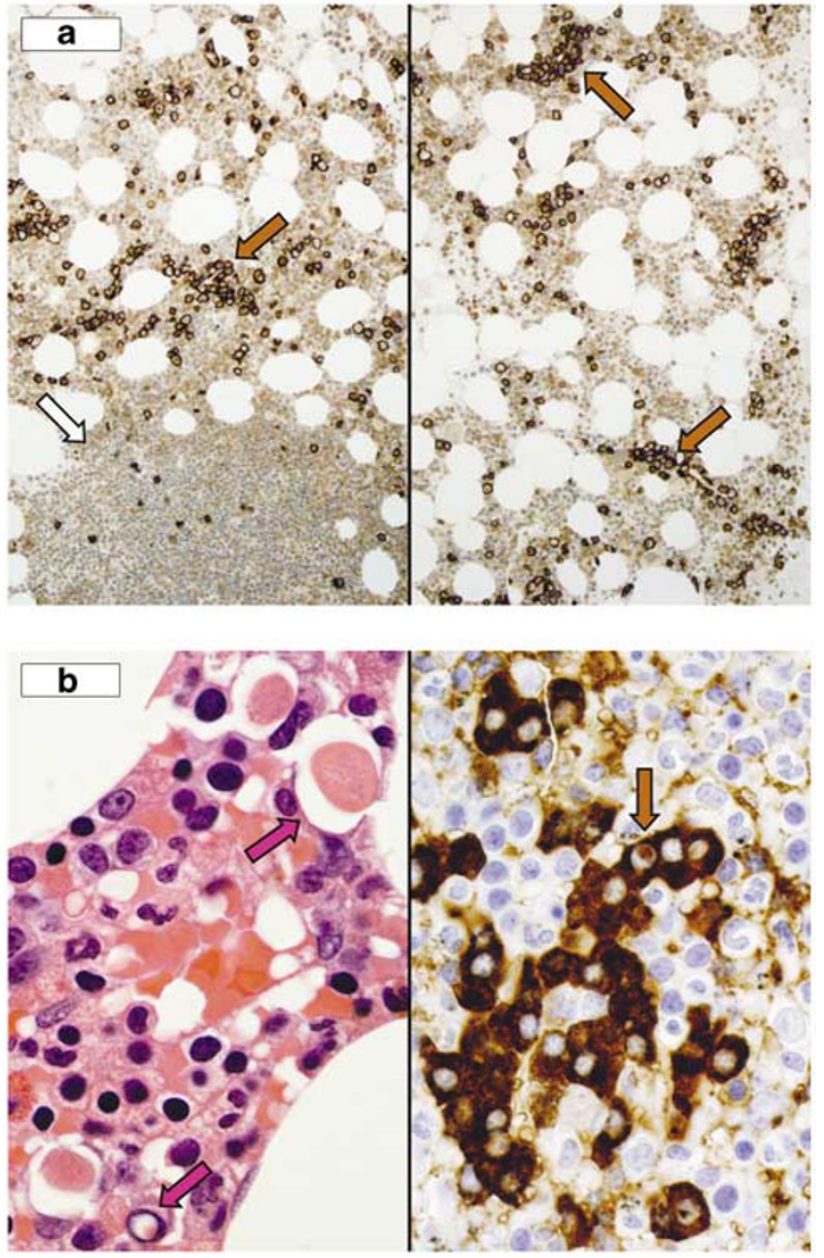

Figure 3 Interstitial plasma cell infiltrates in lymphoplasmacytic lymphoma. Immunohistochemistry reveals aggregates of CD138positive plasma cells in the marrow interstitium (a, left panel, brown arrow) distinct from the nodular lymphoid infiltrate (a, left, white arrow). Aggregates of CD138-positive plasma cells are also present in areas lacking lymphoid infiltrates (a, right panel). The interstitial plasma cells are cytologically atypical with Dutcher bodies and cytoplasmic inclusions (b, left panel, arrows). They show monotypic cytoplasmic immunoglobulin light chain staining (b, right panel, $\lambda$ light chains), which also highlights the Dutcher bodies (arrow). interstitial plasma cell clusters distinct from the lymphoplasmacytic infiltrates, usually with a perivascular distribution (Figure 3a). These interstitial plasma cells were Ig light chain restricted, and frequently contained Dutcher bodies, indicating that they were an element of the neoplastic marrow infiltrates (Figure $3 \mathrm{~b}$ ). In two of the 19 cases, these interstitial plasma cells were the only plasmacytoid element detected by immunohistochemistry.

Lymphocyte analysis by flow cytometry detected surface Ig light chain-restricted B cells in 26 of the 27 cases; in the single-negative case, B-cell infiltrates were identified in the biopsy by immunohistochemistry, and cytoplasmic Ig light chainrestricted B cells and plasma cells were identified by 6-color flow cytometry. None of the monotypic B cells were positive for CD10 or CD103; the other features of these cells are summarized in Table 3 . In most cases the B cells showed moderate or bright surface expression of Ig light chains, CD19 and CD20. In two cases, the B cells were CD20 negative; one of these had a documented history of anti-CD20 monoclonal antibody immunotherapy. CD5 expression by the monotypic $\mathrm{B}$ cells was relatively frequent; ( $43 \%$ of cases) however, most had partial CD5 positivity, characterized by a spectrum of cellular staining ranging from the negative to positive histogram quadrants. In six cases with partial CD5 positivity, CD23 was also partially expressed; in these cases, a diagnosis of chronic lymphocytic leukemia/small lymphocytic lymphoma was excluded by the partial CD5 expression as well as by the bright surface expression of surface Ig and CD20. CD38 expression by the monotypic B cells was frequent, but not universal, with only slightly more than half showing moderate or strong antigen positivity.

A novel single-tube 6-color plasma cell flow cytometry assay, using CD38 and CD138 for cell identification and gating, and analyzing cytoplasmic Ig light chains-CD19 and CD45-was carried out on all 35 marrow aspirates. ${ }^{10}$ In each, a CD38- and

Table 3 Results of B-cell flow cytometric immunophenotyping

\begin{tabular}{|c|c|c|c|c|c|c|c|}
\hline & $S I g^{\mathrm{a}}$ & CD19 & $C D 20^{\mathrm{b}}$ & $C D 22^{\mathrm{b}}$ & $C D 5^{\mathrm{b}}$ & $C D 23^{\mathrm{b}}$ & $C D 38^{\mathrm{b}}$ \\
\hline Antigen positive, number ( $\%$ cases tested) & $26(100)$ & $26(100)$ & $21(91)$ & $23(100)$ & $10(43)$ & $12(52)$ & $16(70 \%)$ \\
\hline Moderate expression $^{\mathrm{c}}$ & 9 & 16 & 12 & 18 & 1 & 2 & 7 \\
\hline Bright expression $^{\mathrm{c}}$ & 11 & 6 & 3 & 0 & 0 & 0 & 2 \\
\hline Dim expression $^{\mathrm{c}}$ & 6 & 4 & 6 & 5 & 0 & 0 & 1 \\
\hline Partial expression ${ }^{\mathrm{C}}$ & 0 & 0 & 0 & 0 & 9 & 10 & 6 \\
\hline
\end{tabular}

\footnotetext{
${ }^{\mathrm{a}}$ Surface immunoglobulin (SIg) light chain.

${ }^{\mathrm{b}}$ Antigen expression was determined in 23 of the 26 cases containing monotypic B cells.

${ }^{\mathrm{c}}$ Pattern of antigen expression by the monotypic B cells: dim, fluorescent midpoint between the first and second histogram decades; moderate, fluorescent midpoint between the second and third histogram decades; bright, fluorescent midpoint near or beyond the third histogram decade; partial, spectrum of staining from negative to positive histogram quadrants (see Morice $e t a l^{12}$ for detailed description).
} 
CD138-positive cell population was identified; however, the proportion of plasma cells estimated by this method was approximately 5-10-fold lower than that estimated by morphological review or by immunohistochemistry (Table 2). In 32 of the 35 cases, the CD38/CD138 copositive cells were cytoplasmic Ig light chain restricted; in the remaining three cases, the plasma cells had polytypic cytoplasmic Ig light chain staining, and therefore the presence of a plasmacytoid disease component was confirmed by immunohistochemistry. In 20 cases, the monotypic plasma cells had the distinctive, bright CD38 and CD138 coexpression typically seen in true plasma cell neoplasms such as multiple myeloma (Figure 4a and c). ${ }^{9,10}$ In contrast to typical true plasma cell neoplasms, however, the plasma cells in lymphoplasmacytic lymphoma were almost always CD19 and CD45 positive. When compared with the cytoplasmic Ig light chain-restricted B cells, which were identified in the same assay by CD45 and side light scatter gating, the plasma cells frequently had a brighter cytoplasmic Ig expression ( 26 of 32 cases) and a dimmer expression of CD19 (19 of 32; Figure 4b). Relatively diminished expression of CD45 by a plasma cell subset was also frequent (Figure $4 \mathrm{~b}$ and $\mathrm{d}$ ). In two cases, the plasma cells identified by 6-color flow cytometry completely lacked CD19 (Figure 4d). In both of these cases, an IgG serum monoclonal paraprotein was present (one was associated with a minor IgM paraprotein), and monotypic B cells were also identified by 4- and 6-color flow cytometry. In four other cases that had a non-IgM isotype serum monoclonal protein, the plasma cells identified by flow cytometry were CD19 and CD45 positive.

The bone marrow findings in the symptomatic and asymptomatic Waldenström's macroglobulinemia patients are compared in Table 4. When comparing these two groups, none of the pathological features were found to discriminate between these two clinically distinct patient cohorts, including the degree of involvement; the degree of plasma cell differentiation detectable by morphology, immunohistochemistry or flow cytometry; and the expression of CD5 by the monotypic B-cells.

\section{Discussion}

The hematopathologist is confronted with making a diagnosis of bone marrow involvement by a B-cell lymphoma with plasmacytic differentiation in a number of settings, including the evaluation of IgM paraproteinemia or potential Waldenström's macroglobulinemia (which tends to be a marrowbased disease), staging of an established lymphoplasmacytic lymphoma (with or without associated Waldenström's macroglobulinemia), and during the evaluation of a newly diagnosed low-grade B-cell malignancy. Although this diagnostic dilemma is relatively commonly encountered, there are few published reports describing the marrow pathology of lymphoplasmacytic lymphoma. ${ }^{5}$ Furthermore, pathological features predicting the presence or absence of associated symptomatic Waldenström's macroglobulinemia are lacking. In an attempt to address these issues, the findings from a group of 35 bone marrows obtained under the first scenario discussed above (ie, as part of the clinical evaluation of potential Waldenström's macroglobulinemia) were studied.

Waldenström's macroglobulinemia/lymphoplasmacytic lymphoma is often described as a neoplasm of 'small lymphocytes, plasmacytoid lymphocytes, and plasma cells', implying a common cytomorphology. Yet, a unifying feature of ours and of earlier studies is that the marrow pathology of lymphoplasmacytic lymphoma is varied both in terms of the pattern of infiltration and the degree of plasmacytic differentiation. ${ }^{1,6,7}$ Given this variability, documenting the presence of both lymphocytic and plasmacytoid/plasmacytic neoplastic cells is fundamental in establishing the diagnosis. In approximately twothirds of our study cases, the histopathological features were consistent with a lymphoplasmacytic lymphoma diagnosis, as the plasma cells were clearly associated with the lymphoid infiltrates, even though they usually represented a minor fraction of the overall cellularity. This morphological impression was supported by immunohistochemistry; the uniform CD138 immunoreactivity of the Waldenström's macroglobulinemia/lymphoplasmacytic lymphoma plasma cells that we observed are in keeping with earlier reported findings. ${ }^{13}$ However, the distribution of the lymphoid infiltrate-associated plasma cells varied between cases, in some being intermingled with the small lymphocytes and in others being arrayed in sheets at the periphery of lymphoid nodules. In a significant subset of cases, the plasma cells were the predominant cell type recognized by morphology and/or immunohistochemistry; the latter because of the strong immunoreactivity of the plasma cells for CD138 immunoreactivity compared with the weak immunoreactivity of the B-lymphocytes for CD20. ${ }^{14}$ In such cases, the lymphoid component of the lymphoplasmacytic lymphoma is difficult to recognize, and immunohistochemistry for other B-cellassociated antigens, such as PAX5 and CD79a, is helpful in revealing the full spectrum of neoplastic cells, and thereby arriving at the correct diagnosis. ${ }^{1,13}$

Another potentially confounding finding highlighted by immunohistochemistry was interstitial plasma cell infiltration distinct from the lymphoid infiltrates that were present in 19 of the 27 cases $(70 \%)$. In two of these cases, these infiltrates represented the only plasma cell component and, therefore, their relationship to the separate lymphoid infiltrates was not readily apparent. In these cases, establishing that the lymphocytes and plasma 

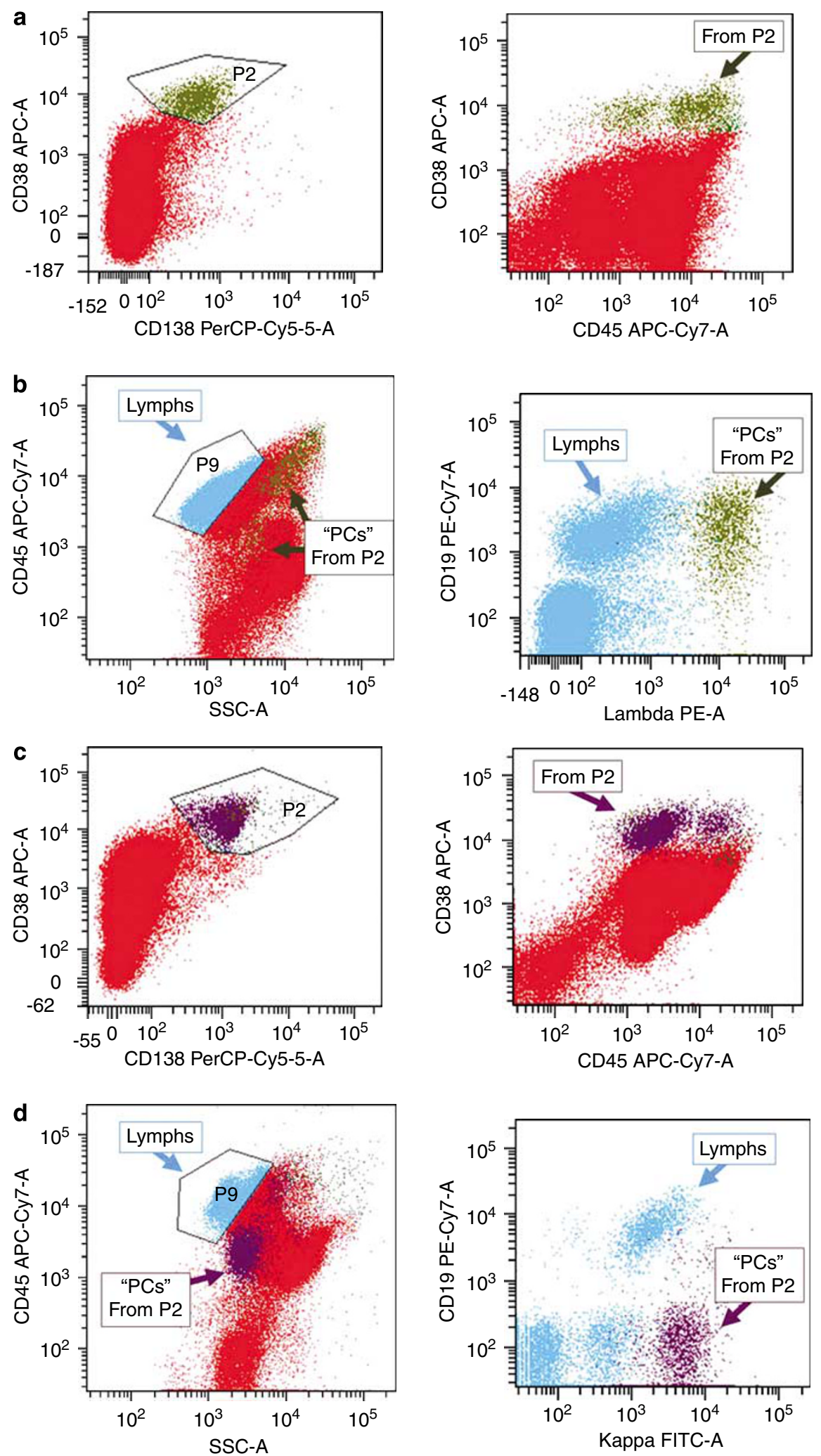

Figure 4 6-color plasma cell flow cytometry in lymphoplasmacytic lymphoma. Plasma cell populations with distinctively bright CD38 and CD138 coexpression identified in two different lymphoplasmacytic lymphoma cases (gate P2, a and c, left panels). These plasma cells show variable CD45 expression (a and $\mathbf{c}$, right panels). Lymphocyte gating by CD45 and side light scatter (b and d, left panels) allows comparison of the B cells and plasma cells from gate P2 (b and d, right panels); the plasma cells typically showed brighter cytoplasmic immunoglobulin light chain and dimmer CD19 and CD45. The PCs in panel c and d completely lacked CD19; in this case, serum protein studies revealed the presence of IgM and IgG monoclonal proteins. 
Table 4 Comparison of findings in symptomatic and asymptomatic Waldenström's macroglobulinemia (WM) cases

\begin{tabular}{|c|c|c|}
\hline & $\begin{array}{c}\text { Symptomatic WM } \\
(\mathrm{n}=29)\end{array}$ & $\begin{array}{c}\text { Asymptomatic WM } \\
(\mathrm{n}=6)\end{array}$ \\
\hline M-Spike, g per $100 \mathrm{ml}$, median (range) & $1.5(0.3-5.1)$ & $1.6(0.4-4.1)$ \\
\hline$\%$ Marrow involvement, median (range) & $30(5-70)$ & $35(10-80)$ \\
\hline \% BM aspirate lymphs by morphology, median (range) & $19(2-72)$ & $25(3-71)$ \\
\hline \% BM aspirate PCs by morphology, median (range) & $1(1-4)$ & $4(1-8)$ \\
\hline \% BM aspirate PCs by FCIP, median (range) & $0.7(0.1-4.6)$ & $0.7(0.3-3.5)$ \\
\hline \% BM biopsy PCs by morphology and IHC, median (range) & $5(1-20)$ & $7(1-20)$ \\
\hline
\end{tabular}

cells were elements of the same disease required showing common Ig light chain expression by both cell types through immunohistochemistry and flow cytometry, and correlation with serum protein electrophoretic studies.

A number of studies have documented the utility of lymphocyte flow cytometry in Waldenström's macroglobulinemia/lymphoplasmacytic lymphoma diagnosis, and yet the reported frequencies of CD5 and CD23 positivity vary widely, ranging from 5 to $20 \%$ and from 1 to $61 \%$, respectively. ${ }^{1,8,13,15}$ Among our cases, CD5 was expressed by $43 \%$ and CD23 by $52 \%$; this frequency of CD5 positivity is nearly identical to that seen in a different group of 16 lymphoplasmacytic lymphoma cases that were recently reported from our institution. ${ }^{12}$ The reasons for the observed differences in Waldenström's macroglobulinemia/lymphoplasmacytic lymphoma CD5 and CD23 expression are unclear; they may be in part related to subtleties in flow cytometry interpretation. In the study citing over $60 \%$ CD23 positivity in Waldenström's macroglobulinemia, only 28 of 75 cases were tested and all 17 positive cases reportedly showed dim antigen expression, raising the possibility that the low levels of positivity observed may not be detected or interpreted as unequivocally positive in other laboratories. ${ }^{15}$ The Waldenström's macroglobulinemia flow cytometry study by San Miguel et $a l^{8}$ reported frequencies of CD5 and CD23 expression closest to those seen in our laboratory. In this study, the authors describe in individual cases a spectrum of antigen expression from negative to positive, similar to that which we observed. Again, in other laboratories, this intraclonal phenotypic heterogeneity may be difficult to detect or not be interpreted as definitively positive. Nevertheless, when considering the current and earlier reports, it is apparent that Waldenström's macroglobulinemia/lymphoplasmacytic lymphoma lacks a pathognomic immunophenotype, although a uniform, strong (moderate-to-bright) expression of CD5 and CD23 in this disease is rare and, when encountered, should prompt consideration of chronic lymphocytic leukemia. Similarly, CD10 positivity in lymphoplasmacytic lymphoma is infrequent; it was not detected in these study cases, although we have encountered occasional CD10-positive cases in the past. ${ }^{1,12}$
Although the Waldenström's macroglobulinemia/ lymphoplasmacytic lymphoma B cells lack a singular immunophenotype, there are a number of findings common to this and other studies. ${ }^{1,8,13,15}$ These include strong surface Ig light chain expression, moderate-to-bright surface CD20 and CD22 expression (all of which help distinguish lymphoplasmacytic lymphoma from chronic lymphocytic leukemia), and variable expression of CD38. Given that plasmacytic differentiation is the hallmark feature of Waldenström's macroglobulinemia/lymphoplasmacytic lymphoma, these features are somewhat unexpected as, in normal B-cell ontogeny, maturation to plasma cells is associated with the downregulation of surface Ig CD20 and CD22 expression, and upregulation of the surface CD38 expression. ${ }^{16}$ A possible explanation for this apparent incongruity is that lymphocyte flow cytometry preferentially detects the disease component most closely resembling the memory B cells from which the neoplasm is putatively derived and does not detect the cells that have undergone plasmacytic differentiation. Regardless of the underlying cause, it is important to note that the phenotype detected by routine B-cell flow cytometry usually does not provide clues to suggest the presence of plasmacytic differentiation in Waldenström's macroglobulinemia/lymphoplasmacytic lymphoma.

Assessment of plasma cells by novel 6-color flow cytometry revealed cytoplasmic Ig light chainrestricted, CD38- and CD138-positive cells in 32 lymphoplasmacytic lymphoma cases, although this method was substantially less sensitive than either morphology or immunohistochemistry in detecting the plasma cell component. Of these 32 cases, 20 had a pattern of the CD38 and CD138 expression virtually identical to that seen in true plasma cell malignancies such as multiple myeloma. ${ }^{10}$ In 18 of these 20 cases, the plasma cells were CD19 positive and the majority expressed CD45, distinguishing them from true plasma cell malignancies that typically lack CD19 and often are predominantly CD45 negative. ${ }^{10}$ These findings closely resemble those reported by San Miguel et al $l^{8}$ in which similar plasma cell antigens were assessed by a 4-color live gating flow cytometry approach in 14 Waldenström's macroglobulinemia cases. Of interest in our study, however, were two lymphoplasmacytic lymphoma 
cases in which the plasma cells lacked detectable CD19. In both cases, an IgG serum paraprotein was present (in one case associated with an IgM paraprotein), but the histology and immunohistochemistry were diagnostic of lymphoplasmacytic lymphoma. Non-IgM paraproteins were detected in six of the study cases; Ig isotype switch in clonal B-cell malignancies with plasmacytic differentiation has been described earlier. ${ }^{17}$ Our findings suggest that the emergence of the non-IgM serum paraprotein is, in some instances, associated with a complete phenotypic transition of a disease component to that of true plasma cell neoplasms. In these two cases with CD19-negative plasma cells, the diagnosis of lymphoplasmacytic lymphoma was still suggested by the coincident presence of cytoplasmic Ig light chain-restricted B cells, but complete understanding of the significance of the 6-color plasma cell flow cytometry results required correlation with the marrow pathology and other clinical and laboratory features, as the data could also lead one to consider the presence of two distinct B-cell and plasma cell neoplasms.

The percentage of plasma cells detected by 6-color flow cytometry was consistently much lower than that detected by histological and/or immunohistochemical examination. This suggests that this method only detects cells sufficiently differentiated toward plasma cells to have become phenotypically distinct, although the alternate possibilities such as poor aspiration of the plasma cells or preferential loss of the plasma cell component during specimen processing for flow cytometry cannot be absolutely excluded. However, when directly comparing these CD38- and CD138-positive cells identified by plasma cell flow cytometry with the B cells in the same analysis tube, the cell populations were clearly distinct, with the plasma cells having brighter cytoplasmic Ig expression and dimmer CD19 and CD45 expression. These features are consistent with plasmacytic differentiation, and the findings support the notion that the B-cell flow cytometry and plasma-cell flow cytometry assays truly recognize distinct cell subsets.

Finally, one goal of these studies was to identify pathological features of lymphoplasmacytic lymphoma, which correlated with the presence or absence of clinical and laboratory features of symptomatic Waldenström's macroglobulinemia. Earlier studies have suggested that the expression of antigens, such as CD5 and CD23, may be correlated with distinctive serological findings. ${ }^{8,15}$ We also postulated that the presence of an increasing proportion of plasma cells detected by morphology, immunohistochemistry, or high-power multicolor flow cytometry may be correlated with increasing symptomatic paraproteinemia, as these cells would presumably be the most active Ig secretors. Unfortunately, no pathological predictors of the presence or absence of symptomatic Waldenström's macroglobulinemia were identified. Indeed, the only laboratory correlate of a pathological feature in the Waldenström's macroglobulinemia cases was the presence of a non-IgM paraprotein in cases containing CD19-negative plasma cells. The power of our study in this regard may be limited by the relatively small number of cases; however, the findings may also indicate that the paraprotein is produced by the full spectrum of neoplastic cells and not by an isolated morphologically or phenotypically distinct subset.

In conclusion, although the bone marrow features of lymphoplasmacytic lymphoma vary, in many cases, the disease process can be recognized morphologically. Frequently, however, the diagnosis is not histologically evident owing either to a predominance of plasma cells or to physical dissociation of the lymphocytic and plasmacytic components. In such a case, immunophenotyping by immunohistochemistry and/or flow cytometry is useful in identifying the full spectrum of cellular elements and thereby establishing the diagnosis. It is noteworthy, however, that routine B-cell flow cytometry often does not suggest the presence of a plasmacytic differentiation. Conversely, 6-color flow cytometry is relatively insensitive in detecting the plasma cell component and, in this assay, the lymphoplasmacytic lymphoma plasma cells may closely resemble those of true plasma cell neoplasms. For these reasons, when performing plasma cell-flow cytometry in potential lymphoplasmacytic lymphoma cases, particular attention should be paid to the expression of CD19 and CD45, as well as to the presence of cytoplasmic Ig light chain-restricted B cells, and the results must be correlated with the marrow pathology and laboratory features. Furthermore, although immunophenotyping allows for a detailed characterization of the plasmacytic and lymphocytic disease components, it does not predict for the presence or absence of symptomatic Waldenström's macroglobulinemia. Finally, although this study exclusively focused on lymphoplasmacytic lymphoma and correlations with the presence of symptomatic Waldenström's macroglobulinemia, it remains unknown whether this multifaceted approach can reveal features that distinguish lymphoplasmacytic lymphoma from marrow involvement in other B-cell lymphoma with plasmacytic differentiation. The authors hope to address this question in future studies.

\section{Acknowledgements}

We thank our Mayo Clinic hematology colleagues, especially members of the Myeloma, Amyloidosis, and Dysproteinemia Disease-Oriented Group, for their open collaboration and dedication to patient care. The authors also thank Cece Meyers, Laura Schroeder, and the Cell Kinetics laboratory for providing outstanding service in clinical flow cytometry. 


\section{Disclosure}

The authors have no conflict of interest to disclose.

\section{References}

1 Remstein ED, Hanson CA, Kyle RA, et al. Despite apparent morphologic and immunophenotypic heterogeneity, Waldenstrom's macroglobulinemia is consistently composed of cells along a morphologic continuum of small lymphocytes, plasmacytoid lymphocytes, and plasma cells. Semin Oncol 2003;30: 182-186.

2 Lin P, Hao S, Handy BC, et al. Lymphoid neoplasms associated with IgM paraprotein: a study of 382 patients. Am J Clin Pathol 2005;123:200-205.

3 Jaffe ES, Harris NL, Stein H, et al. (eds). Pathology and Genetics: Tumours of Haematopoietic and Lymphoid Tissues. IARC Press: Lyon, 2001.

4 Waldenstrom J. Incipient myelomatosis or 'essential' hyperglobulinemia with fibrinoginopenia-a new syndrome? Acta Med Scand 1944;117:216-247.

5 Owen RG, Treon SP, Al-Katib A, et al. Clinicopathological definition of Waldenstrom's macroglobulinemia: consensus panel recommendations from the Second International Workshop on Waldenstrom's Macroglobulinemia. Semin Oncol 2003;30:110-115.

6 Andriko JA, Aguilera NS, Chu WS, et al. Waldenstrom's macroglobulinemia: a clinicopathologic study of 22 cases. Cancer 1997;80:1926-1935.

7 Arber DA, George TI. Bone marrow biopsy involvement by non-Hodgkin's lymphoma: frequency of lymphoma types, patterns, blood involvement, and discordance with other sites in 450 specimens. Am J Surg Pathol 2005;29:1549-1557.
8 San Miguel JF, Vidriales MB, Ocio E, et al. Immunophenotypic analysis of Waldenstrom's macroglobulinemia. Semin Oncol 2003;30:187-195.

9 de Tute RM, Jack AS, Child JA, et al. A single-tube sixcolour flow cytometry screening assay for the detection of minimal residual disease in myeloma. Leukemia 2007;21:2046-2049.

10 Morice WG, Hanson CA, Kumar S, et al. Novel multiparameter flow cytometry sensitively detects phenotypically distinct plasma cell subsets in plasma cell proliferative disorders. Leukemia 2007;21:2043-2046.

11 Kurtin PJ, Hobday KS, Ziesmer S, et al. Demonstration of distinct antigenic profiles of small B-cell lymphomas by paraffin section immunohistochemistry. Am J Clin Pathol 1999;112:319-329.

12 Morice WG, Kurtin PJ, Hodnefield JM, et al. Predictive value of blood and bone marrow flow cytometry in Bcell lymphoma classification: comparative analysis of flow cytometry and tissue biopsy in 252 patients. Mayo Clin Proc 2008;83:776-785.

13 Owen RG, Barrans SL, Richards SJ, et al. Waldenstrom macroglobulinemia. Development of diagnostic criteria and identification of prognostic factors. Am J Clin Pathol 2001;116:420-428.

14 Kyle RA, Remstein ED, Therneau TM, et al. Clinical course and prognosis of smoldering (asymptomatic) multiple myeloma. N Engl J Med 2007;356:2582-2590.

15 Konoplev S, Medeiros LJ, Bueso-Ramos CE, et al. Immunophenotypic profile of lymphoplasmacytic lymphoma/Waldenstrom macroglobulinemia. Am J Clin Pathol 2005;124:414-420.

16 Uckun FM. Regulation of human B-cell ontogeny. Blood 1990;76:1908-1923.

17 Sahota SS, Garand R, Bataille R, et al. VH gene analysis of clonally related IgM and IgG from human lymphoplasmacytoid B-cell tumors with chronic lymphocytic leukemia features and high serum monoclonal IgG. Blood 1998;91:238-243. 